\title{
Hadronic Masses and Regge Trajectories
}

\author{
Silvana Filipponi $[1,2]$ and Yogendra Srivastava ${ }^{\dagger}[2,3]$
}

September 6, 2018

\author{
HUTP-97/A093 \\ 1.Physics Department, Harvard University, Cambridge MASS, USA. \\ 2.Dipartimento di Fisica e INFN, I-06100 Perugia Italy. \\ 3.Physics Department, Northeastern University, Boston MASS, USA.
}

\begin{abstract}
A comprehensive phenomenological analysis of experimental data and some theoretical models is presented here (for mesons) to critically discuss how Regge trajectory parameters depend on flavor. Through analytic continuation of physical trajectories (obtained from resonance data) into the space like region, we derive the suppression factor for heavy flavor production. The case of our D Regge exchange, both for $\mathrm{D}$ and $\Lambda_{c}$ production, is considered in some detail. Good agreement with data is reached confirming that indeed the slopes of heavier flavors decrease. This result suggests that the confinement potential has a substantial dependence on the quark masses. In a simple non-relativistic model, constrained to produce linear Regge trajectories, it is shown that a linear quark mass dependence is required (in the confinement part of the potential) in order for the slope to decrease in the appropriate way.
\end{abstract}

\section{Introduction}

Hadron internal dynamics is described by QCD but in deriving their properties many problems arise essentially due to the mathematical complexity of relativistic bound state problems in non abelian gauge theories. This has led to techniques and approximation schemes to perform phenomenological calculations which differ according to the species of the hadron. Since QCD is flavor independent but the hadrons are not, it is only natural that quark masses which distinguish flavor appear as crucial quantum numbers not only in fixing the scale of the hadronic masses but in determining the choice of the approximation itself.

*Silvana@pg.infn.it

†Srivastava@pg.infn.it 
A relativistic or non-relativistic model is usually invoked depending upon whether the ratio $\frac{m}{\Lambda_{\chi}}<1$ or to $>1$, where $\Lambda_{\chi}$ is the hadronic scale and $m$ the quark mass. It would then appear that the dynamics for light quarks should be largely detemined by $\Lambda$ and by $m$ for heavy flavors. The case of heavylight systems would lie somewhere in between. The purpose of this work is to investigate the question of quark mass dependence in a systematic way.

Our analysis is based on the construction of Regge trajectories under the the following two hypotheses. First, we assume all trajectories to be linear in the squared mass (of the hadronic state) for all flavours.

$$
\alpha(s)=\alpha(0)+s \alpha^{\prime}
$$

For light baryons and mesons, we have ample and compelling phenomenological evidence supporting this assumption. Our analysis finds no evidence for strong deviations from linearity also for heavier flavors.

The second assumption is that the functional dependence of the two parameters $\alpha(0)$ and $\alpha^{\prime}$ on quark masses is through the combination $\left(m_{1}+m_{2}\right)$. Arguments in support of this hypothesis are presented in section two.

In section three through an analysis of Regge trajectories for mesons of all flavors, we obtain analytic forms for the slope as well as the intercept parameters as a function of $\left(m_{1}+m_{2}\right)$. The "distances" between trajectories are also investigated in the same fashion. In section four trajectories are constructed on which physical mesons lie. In section five, we employ their analytic continuation into the space like region to discuss how heavy flavour production is suppressed. The case of D meson trajectory exchange is analysed in some detail. Our model predictions are compared successfully with available experimental data. In section six we address the implications which our Regge trajectories may have on the dynamics underneath. The decrease in the slope for heavier flavors is argued to imply that the confining potential is strongly flavor dependent. A non-relativistic potential model exhibiting linear trajectories is constructed where a simple linear dependence on the quark mass (for the confinement term) reproduces the appropriate behavior in the slope.

\section{Choice of the variable $\left(m_{1}+m_{2}\right)$}

Let us consider a meson of mass $M\left(m_{1}, m_{2}\right)$ as a bound state of a quark of mass $m_{1}$ and an anti-quark of mass $m_{2}$. Quite generally, M may be parametrized as a function of $\rho=\sqrt{m_{1} m_{2}}$ and $\tilde{m}=\left(m_{1}+m_{2}\right)$. Since quarks have never been observed as free particles, there is no unique definition of $m_{1}$ and $m_{2}$. Transcending the bound state dynamics, this uncertainty further complicates how $M$ depends on $\rho$ and $\tilde{m}$. For the constituent quark picture adopted here, we present arguments below in favor of $\tilde{m}$ being the relevant parameter upon which $M$ depends, for practically all cases where data are available. In some 
cases, where the dependence on $\rho$ turns out to be small but not negligible, it may be incorporated perturbatively.

As a widely accepted convention, quarks are considered light if $m<\Lambda_{\chi}$ $(\mathrm{u}, \mathrm{d}, \mathrm{s})$ and heavy if $m>\Lambda_{\chi}(\mathrm{c}, \mathrm{b}, \mathrm{t})$, indicating that very different properties arise depending on this scale $\left(\Lambda_{\chi} \approx 1 \mathrm{GeV}\right)$. Given the above classification for quark masses, there are four types of mesons: (i) both quarks are light; (ii) both quarks have the same mass; (iii) one quark is light and the other heavy; (iv) both quarks are heavy. For case (i), both $\rho$ and $\tilde{m}$ are much smaller than $\Lambda_{\chi}$, hence negligible in our scale for all quark masses. For case(ii), $\rho$ and $\tilde{m}$ degenerate into a single parameter. For case (iii), $\tilde{m}$ is the only remaining parameter. Hence, the question whether $\tilde{m}$ is the dominant parameter is of relevance only for case (iv). For the subclass of (iv) which can be described via non-relativistic dynamics, the dependence on $\rho$ (or, equivalently on the reduced mass) must by implication be small. In such cases, it can be included perturbatively. For the rest of class (iv), the dependence on $\rho$ cannot a priori be guaranteed to be small. In practical terms, this may be quite relevant only for the $(b \bar{c})$ system, where appreciable differences in masses may occur in parametrizations with or without the inclusion of terms containing $\rho$. Thus, data on this system would be crucial in distinguishing between the two choices.

All the analysis of the next section will be performed using the $\tilde{m}$ variable. Without the above justification, the same choice has also been made in [1]. Corrections due to electric charge or isospin are not included here.

\section{The phenomenological analysis}

\subsection{The $\alpha^{\prime}$ and $\alpha_{I}(0)$ parameters of the Regge trajectories}

In this section, we derive analytic forms for the slope and intercept parameters for the Regge trajectories for all mesons. For this purpose, we use experimental data [2] as well as results from theoretical models. Theoretical information is required to supplement the experimental one for mesons composed of heavy quarks where only the lowest part of the spectra has been measured. We selected some models which fit the measured energy levels and predict the masses for higher resonances. For some states, quark model needs to be invoked to fix the spin [2], whenever it has not been determined experimentally. The spin quantum number being crucial for our analysis, it is essential to verify whether the quark model prediction is confirmed by other independent calculations. As experimental spectra are almost unkown for the B mesons and poorly measured for the D mesons, we do not attempt for them the same detailed analysis as has been done for charmonium and bottomium. For the D system, only a rough determination of the Regge trajectories can be made from experimental data.

While using experimental data, our choice for the quark masses are as fol- 
lows:

$$
m_{u}=m_{d}=0.03 \mathrm{GeV}, \quad m_{s}=0.5 \mathrm{GeV}, m_{c}=1.7 \mathrm{GeV} \text { and } m_{b}=5 \mathrm{GeV} .
$$

While using inputs from theoretical models, values for quark masses are fixed according to the ones used in the particular model.

Let us begin with the slope parameter. In Fig.1 input data for the slopes are presented.

For light mesons, we refer to a recent analysis of Regge trajectories in [3], and to the MIT bag model «] which gives the theoretical value for $\alpha_{b a g}^{\prime} \approx 0.9 \mathrm{GeV}^{-2}$. In this model, the bag is supposed to be a rapidly rotating, linearly extended object, where the massless quarks are fixed at the ends; the slope parameter is obtained through a classical calculation of the energy and angular momentum of the bag.

The four experimental points (the bullets) for light mesons, have been determined analyzing leading Regge trajectories for systems with different isospin (along with their quark content):

$\mathrm{I}=0$, both $\omega(u \bar{u}+d \bar{d}$ bound states $)$ and $\phi(s \bar{s}$ bound states $)$ resonances- with slightly different slopes;

$\mathrm{I}=1, \rho$ resonances;

$\mathrm{I}=1 / 2, \mathrm{~K}$ resonances.

For charmonium, we calculate the slope parameter using the masses of the two states that have been experimentally measured both for the leading $[J / \psi(1 s)-$ $\left.\chi_{c 2}(1 p)\right]$ and for the second trajectory $\left[\eta_{c}(1 s)-\chi_{c 1}(1 p)\right]$ which differ by one unit of spin. A similar situation is found for bottomium. For both these systems a more complete spectrum is provided by theoretical models $([\sqrt{5}],[6],[7])$. We check that an almost linear behaviour is reproduced with the predicted masses for the higher J states. For both systems, the calculated slopes are very close to the ones derived from our analysis. This is not an obvious result because the model predictions for the unkown $\mathrm{J}$ states are given in a completely independent way without imposing any linear behaviour. The theoretical models we analyse are non relativistic potential models (relativistic correction terms are included by hand) where a central potential describes the interaction. In [5] a smooth function interpolating between the Coulomb and the linear term fits the spectra. The other choices for the potential are: $V(r)=A+B r^{0.1}$ for [6] and $V(r)=$ $\tilde{A}+\frac{\tilde{B}}{r^{0.1}}$ for [7]. For these three models, the slopes are calculated and included in our analysis.

Experimental data for the $\mathrm{D}$ and $D_{s}$ mesons are used to obtain their slope. Predictions from a semirelativistic model in [8] for the D and B mesons are also included.

All the inputs described above have been used in our analysis and are shown in Fig.1, along with a global fit. The analytic behaviour for the slope is found to be

$$
\alpha^{\prime}=\frac{0.9}{1+0.2\left(\frac{m_{1}+m_{2}}{G e V}\right)^{3 / 2}} G e V^{-2}
$$


An identical analysis has also been carried out for $\alpha_{I}(0)$, where the subscript I refers to the leading Regge trajectory. All input data points are shown in Fig.2 along with the following analytic expression

$$
\alpha_{I}(0)=0.57-\frac{\left(m_{1}+m_{2}\right)}{G e V}
$$

In Fig.2 only two entries (they refer to the $B_{c}$ system) have too small a value compared to the general trend. We are unable to explain these deviations from the expected behaviour.

Before concluding this section, we consider some consequences following from our result for the slope parameter, eq.3. The $3 / 2$ power implies a rather large value for the kinetic energy possessed by bound state of heavy quarks. This becomes substantial already for the $B_{c}$ system, and is certainly so for toponium and top mesons. Unfortunately, mass spectra are completely unkown in the first case [9], while top bound states are predicted neither to form, due to the large mass and short life time (through weak decays) of the top quark. Presently, it is not possible to verify from data whether such a large kinetic energy term exists.

It must be emphasized that parametrizations which include other quark mass dependences than $m_{1}+m_{2}$ alone, might change significantly the $3 / 2$ power law. The accuracy of present data renders difficult such an analysis in terms of more than one combination of the quark masses.

In string type models [10], where the associated kinetic energies are not overly large, $1 / \alpha^{\prime}$ would be linear in $\tilde{m}=m_{1}+m_{2}$ (within our assumption that $\alpha^{\prime}$ only depends upon $\tilde{m}$ ). Hence, we tried to constrain the fit to reproduce this behavior. A phenomenological problem arises however with the light sector. Since, $\alpha^{\prime}=\frac{\alpha^{\prime}(0)}{1+A \tilde{m}}$ has a large negative derivative for small $\tilde{m}$, there appear significant variations for the slope in that region. On the other hand, we require for all the slopes of the light sector- $\alpha_{\text {light }}^{\prime} \approx(0.8 \div 0.9) \mathrm{GeV}^{-2}$. Such can be obtained only with an almost perfect degeneracy in the $\mathrm{u}, \mathrm{d}$ and s quark masses.

We hope to return to this interesting but delicate question elsewhere. Below we continue our analysis with the unconstrained fit, eq.3, which reproduces the observed fall off in the slope quite well.

\subsection{Level splitting through the $\alpha(0) \equiv \alpha_{I}(0)-\alpha_{I I}(0)$ param- eter.}

Let us now consider the spacing between the first and successive trajectories for various mesons. This distance is defined to be the energy squared gap between states of fixed $\mathrm{J}$ for successive trajectories, $\left[E_{J}^{I+1}\right]^{2}-\left[E_{J}^{I}\right]^{2}$. We also analyse the corresponding energy level splitting $\left[E_{J}^{I+1}\right]-\left[E_{J}^{I}\right]$.

There is phenomenological evidence suggesting that successive trajectories alternate between states of normal and abnormal parity. That is, the first 
trajectory contains the normal set starting with $J^{P}=1^{-}$, followed by the abnormal set with $J^{P}=0^{-}$, and so on. In particular, lowest $\mathrm{J}=1$ states for any meson [2] has negative parity. As energy increases, we encounter a state of positive parity, followed by a negative parity state and so on. This trend is summerized in Table I.

\begin{tabular}{|l|l||l|l||l|l||l|l||}
\hline \multicolumn{1}{|c|}{ J $\mathbf{1}$ states } \\
\hline \hline State & $\mathrm{P}$ & State & $\mathrm{P}$ & State & $\mathrm{P}$ & State & $\mathrm{P}$ \\
\hline \hline$\omega(782)$ & - & $h_{1}(1170)$ & + & $\omega(1420)$ and $\omega(1600)$ & - & $?$ & + \\
$\rho(770)$ & - & $b_{1}(1235)$ & + & $\rho(1450)$ & - & $?$ & + \\
$K^{*}(892)$ & - & $K_{1}(1270)$ & + & $K^{*}(1410)$ & - & $K_{1}(1650)$ & + \\
$\phi(1020)$ & - & $h_{1}(1382)$ & + & $\phi(1680)$ & - & $?$ & + \\
\hline$D^{*}$ & - & $D_{1}(2420)$ & + & $?$ & - & $?$ & + \\
$D_{s}^{*}$ & - & $D_{1 s}(2536)$ & + & $?$ & - & $?$ & + \\
\hline$J / \psi(1 s)$ & - & $\chi_{c 1}(1 p)$ & + & $\psi(2 s)$ and $\psi(3770)$ & - & $?$ & + \\
\hline$Y(1 s)$ & - & $\chi_{b 1}(1 p)$ & + & $Y(2 s)$ & - & $\chi_{b 1}(2 p)$ & + \\
\hline
\end{tabular}

Table I: Spectrum of spin one states.

Exceptions to this regularity are present and we discuss them briefly in the following. For example, for the omega resonances, we have two closely lying states, $\omega(1420)$ and the $\omega(1600)$, which have both been assigned negative parity. A similar situation is found for the charmonium states, $\psi(2 s ; 3686.00 \pm 0.09 \mathrm{MeV})$ and $\psi(3770)$ states.

Another possible exception concerns the $\hat{\rho}(1405), J^{P}=1^{-}$state, which was observed but not reconfirmed. In fact this state is omitted from summary table, even if reported in [2]. If such a state did exist, it would be out of the scheme presented above.

On the other hand, for $\mathrm{K}$ mesons the situation is extremely regular. First three states follow this pattern. Then somewhat doubtful K(1650) of positive parity is followed by $K^{*}(1680)$ of negative parity. This last entry is not shown in the previous table for lack of space.

As can be seen in Table I, data are scarce for D mesons. In any case, the known states follow the regular pattern and we have included them in our analysis. For the first entry on line 6 of Table I, the state labelled $D_{s}^{*}$, quantum numbers have not yet been measured. The assignment $J^{P}=1^{-}$for this state appears consistent with the general pattern. Hence, we have included it in the first column.

Finally for the charmonium and bottomium systems, beyond the tabulated states, a series of unconfirmed $\mathrm{P}=-$ states have been reported $[\psi(3770)$, $\psi(4040), \psi(4160), \psi(4415)$ for charmonium and Y(4s), Y(10860), Y(11020) for bottomium]. Apart from these possible exceptions, the alternating behaviour between normal and abnormal sets of Regge trajectories seem to be confirmed. 
In Fig.3, energy splitting and the energy squared gap between spin one states from columns I and II of Table I are plotted as a function of $m_{1}+m_{2}$. No input from theoretical models are available since only normal states were considered there.

As a general behaviour, in Fig. 3 energy splitting seems to be almost constant around $0.4 \mathrm{GeV}$ for all existing mesons, while energy squared increases.

The distance between the normal and the successive abnormal trajectory is defined through the energy squared gap for a fixed J

$$
\left[\bar{E}_{J}\right]^{2}-\left[E_{J}^{I}\right]^{2}=\frac{1}{\alpha^{\prime}}\left[\alpha_{I}(0)-\bar{\alpha}(0)\right] .
$$

The energy splitting is given by

$$
\bar{\Delta} E_{J} \equiv \bar{E}_{J}-E_{J}^{I}=\frac{1}{\sqrt{\alpha^{\prime}}}\left\{\sqrt{J-\bar{\alpha}(0)}-\sqrt{J-\alpha_{I}(0)}\right\}
$$

where the tilde superscript refers to the abnormal and the index I to the leading trajectory. From Fig.3, we find that $\bar{d} \alpha(0) \equiv \alpha_{I}(0)-\bar{\alpha}(0)$ depends weakly upon $m_{1}+m_{2}$ and this dependence has been ignored. A conservative estimate for this parameter is given by

$$
0.6<\bar{d} \alpha(0) \equiv \alpha_{I}(0)-\bar{\alpha}(0)<1.1
$$

For $\alpha^{\prime}$ and $\alpha_{I}(0)$ we use our analytic results given in eq 3 and eq. 1 to obtain the continuous curves shown in Fig.3. We repeat the same analysis for the distance between the leading and the second trajectory, using both the experimental data (columns I and III from Table I) and theoretical estimates from models discussed before. In Fig.4, we plot the energy squared gap and, in Fig.5, the energy gap between the leading and the second trajectory, as a function of $m_{1}+m_{2}$. From these curves, we deduce

$$
d \alpha(0) \equiv \alpha_{I}(0)-\alpha_{I I}(0) \approx(1.3 \div 1.6)
$$

These data, even though not very precise, seem to indicate that the separations between the leading normal and the successive abnormal and between the abnormal and the second trajectories are not equal.

\section{Leading trajectories for heavy mesons}

In section three we derived how Regge parameters functionally depend on $m_{1}+$ $m_{2}$. As a function of the sum of the constituent quark masses and the rest mass of the corresponding bound state, the leading trajectory is found to be

$$
J\left(m_{1}+m_{2}, M^{2}\right)=0.57-\frac{\left(m_{1}+m_{2}\right)}{G e V}+\frac{0.9 G e V^{-2}}{1+0.2\left(\frac{m_{1}+m_{2}}{G e V}\right)^{3 / 2}} M^{2}
$$


For $m_{1}+m_{2} \approx 10 \mathrm{GeV}$ the slope of the corresponding trajectory (describing bottomium) is close to zero.

The effective $m_{1}+m_{2}$ parameter for any meson is derived inserting into eq. 9 the experimental value for the mass of the lowest energy, $J=1$ state. The precision of this analysis is estimated to be of order of ten percent. At the present level, eq.9 cannot be used to "deduce" the quark masses.

About the $D_{s}$ and $B_{s}$ mesons, the lowest energy $\mathrm{J}=1$ state has not yet been detected, [2]. As possible candidates, we choose $D_{s}^{*}=(2112.4 \pm 0.7) \mathrm{MeV}$ (the same choice has been made in section three) and $B_{s}^{*}=(5416.3 \pm 3.3) \mathrm{MeV}$. These states are used here to derive the slope and intercept parameters for $D_{s}$ and $B_{s}$ systems.

This tecnique has been used to calculate rest mass spectra. The "mass formula", as well as results for D and B mesons, charmonium and bottomium spectra are presented in another paper [11. In Table II, the slope and intercept parameters have been calculated for all the heavy mesonic sector, except for the $B_{c}$ system, whose ground state has not yet been detected [9], and toponium and top mesons (see [11] for a detailed discussion about mesons composed of the top quark and their Regge trajectories).

\begin{tabular}{|l||ll|}
\hline Meson & $\alpha_{I}(0)$ & $\alpha^{\prime}\left(\mathrm{GeV}^{-2}\right)$ \\
\hline \hline $\mathrm{D}$ & -1.35 & 0.59 \\
$D_{s}$ & -1.51 & 0.56 \\
Charmonium & -2.83 & 0.40 \\
$\mathrm{~B}$ & -5.45 & 0.23 \\
$B_{s}$ & -5.55 & 0.22 \\
Bottomium & -9.67 & 0.12 \\
\hline
\end{tabular}

Table II:Our results for the slope and intercept parameters of heavy mesons.

About the slope parameter, we find a very good agreement with independent calculation in [1], which gives $1 / \alpha^{\prime}=2.48 \mathrm{GeV}^{2}$ for charmonium and $1 / \alpha^{\prime}=$ $6.21 \mathrm{GeV}^{2}$ for bottomium.

In Fig. 6 our leading trajectories for all existing flavours are plotted to show the intersection region. About the light sector, that has not been analysed in this work, only one trajectory is drawn for all isospin and the corresponding parameters are calculated for $m_{1}+m_{2}=0$.

\section{Predictions for the space-like region}

Up to now our analysis has been concerned with physical states lying on the trajectories. Of course, Regge theory allows for applications in the space-like region through predictions for hadronic scattering processes, since its asymptotic behaviour in energy is related to the exchanged Regge trajectory. Exchanges of 
light trajectories for exclusive processes have been quite successful in the past. For the heavy sector, only inclusive data are available for charm production. We employ the analytic continuation of the D Regge trajectory to compute the inclusive production of $\mathrm{D}$ mesons and $\Lambda_{c}$ baryon through the di-triple Regge formalism. These results are compared with available experimental data.

Such an application to the space-like region is a completely independent check for our constructed Regge trajectories since other data and a different part of the phase space are now involved. Similar data are not available for the $\mathrm{B}$ system, thus only theoretical predictions are given.

\subsection{The suppression factor for heavy flavour production}

For an inclusive reaction $a+b \rightarrow c+X$, Regge theory predictions are given in the Tri-Regge asymptotic limit, [12], where $t=\left(p_{a}-p_{c}\right)^{2}$ is kept fixed, both the c.m. energy $s=\left(p_{a}+p_{b}\right)^{2}$ and $M_{X}^{2}=\left(p_{a}+p_{b}-p_{c}\right)^{2}$ are large, but $M_{X}^{2} / s$ is small. For an inclusive process the differential cross section is given by, 13.

$$
\frac{d^{2} \sigma}{d M_{X}^{2} d t} \approx \frac{\gamma_{0}(t)}{s}\left(\frac{M_{X}^{2}}{s}\right)^{1-2 \alpha(t)}
$$

where $\alpha(t)$ is the Regge trajectory for the exchanged particle. Momentarily neglecting the t dependence, we have

$$
\frac{d \sigma}{d M_{X}^{2} / s} \approx\left(\frac{s}{M_{X}^{2}}\right)^{-n} \quad n=1-2 \alpha(0)
$$

showing the energy suppression factor in the differential cross section for inclusive heavy flavour production. The power $\mathrm{n}$ is related to $\alpha(0)$, hence it linearly increases with the sum of the quark masses constituting the meson. Thus, according to eq. 4 the suppression factor becomes larger as one goes from the D meson to bottomium. Predictions for all heavy flavor sectors are gived in Table III.

\begin{tabular}{|c|c|}
\hline Meson & $\mathrm{n}$ \\
\hline \hline $\mathrm{D}$ & 3.7 \\
$D_{s}$ & 4.0 \\
Charmonium & 6.7 \\
$\mathrm{~B}$ & 12 \\
$B_{s}$ & 12 \\
Bottomium & 20 \\
\hline
\end{tabular}

Table III: The power $n$ controlling the suppression term, eq.11, appearing in the differential cross section for heavy flavor production. 


\subsection{Inclusive charm production.}

The Fermilab experiment E769, 14, has detected charged and neutral D mesons through $250 \mathrm{GeV} \pi^{-} N$ interactions, using targets of $\mathrm{Be}, \mathrm{Al}, \mathrm{Cu}, \mathrm{W}$. There the differential cross section for charm meson production is analyzed using the Feynman-x $\left(x_{F}\right)$ and transverse momentum $\left(p_{T}^{2}\right)$ variables. The following fit has been proposed in the factorized form for the range $0.1<x_{F}<0.7,(0<$ $\left.p_{T}^{2}<4 \mathrm{GeV}^{2}\right)$

$$
\frac{d^{2} \sigma}{d x_{F} d p_{T}^{2}} \approx\left(1-x_{F}\right)^{n} e^{-b p_{T}^{2}}
$$

The same interaction was investigated at CERN in two different experiments (NA27, 15 and NA32, 16]) using different targets, beam energy and ranges for the $x_{F}$ and $p_{T}^{2}$ variables. Particulars for all three experiments are shown in Table IV below, along with the best values for $\mathrm{n}$ and $\mathrm{b}$. As we can see all three independent measurements give practically the same result for both parameters.

\begin{tabular}{|l|l|l|l|}
\hline Expt. & E769 & NA32 & NA27 \\
\hline \hline$p_{\text {beam }}(\mathrm{GeV})$ & 250 & 230 & 360 \\
Target & $\mathrm{Be}, \mathrm{Al}, \mathrm{Cu}, \mathrm{W}$ & $\mathrm{Cu}$ & $\mathrm{H}$ \\
\hline \hline$x_{F}$ fit range & 0.1 to 0.7 & 0.0 to 0.8 & 0.0 to 0.9 \\
\hline $\mathrm{n}$ & $3.9 \pm 0.3$ & $3.74 \pm 0.23 \pm 0.37$ & $3.8 \pm 0.63$ \\
\hline \hline$p_{T}^{2}$ fit range $\left(\mathrm{GeV}^{2}\right)$ & 0 to 4 & 0 to 10 & 0 to 4.5 \\
\hline $\mathrm{b}\left(\mathrm{GeV}^{-2}\right)$ & $1.03 \pm 0.06$ & $0.83 \pm 0.03 \pm 0.02$ & $1.18 \pm 0.18$ \\
\hline
\end{tabular}

Table IV:Values of $n$ and $b$ for all three experiments.

A similar value for $\mathrm{n}\left(n=3.69_{-0.71}^{+0.74}\right)$ is obtained in [17] for the charmed baryon $\Lambda_{c}^{+}$production from $230 \mathrm{GeV} \pi^{-} \mathrm{Cu}$ and $K^{-} \mathrm{Cu}$ interactions.

Both in $\pi^{-} N \rightarrow D X$ and $\pi^{-} N \rightarrow \Lambda_{c} X$, a D trajectory is exchanged; Fig.7 represents this exchange for the $\pi N \rightarrow D X$ reaction.

In order to test our predictions from Table III, we define the ratio between theoretical and experimental cross section as follows

$$
R\left(M_{X}^{2}, t\right)=\frac{\left[d^{2} \sigma / d M_{X}^{2} d t\right]_{T H E O}}{\left[d^{2} \sigma / d M_{X}^{2} d t\right]_{E X P}},
$$

where the numerator is given in eq.10. For the denominator, eq.12 must be tranformed from the $\left(x_{F}, p_{T}^{2}\right)$ into the $\left(M_{X}^{2}, t\right)$ variables and calculated in the Tri-Regge limit. The transformation along with some other kinematic details are given in the appendix, where we also show that $M_{X}^{2} / s \approx 0.3 \div 0.6$ (or equivalently $x_{F} \approx 0.4 \div 0.7$ ) is the reliable region for testing our predictions.

Thus in the triple-regge limit, for a fixed $t$ value, the ratio $\mathrm{R}$ is given by

$$
\left.R\left(\frac{M_{X}^{2}}{s}\right)\right|_{t} \approx \frac{\left(1-\frac{M_{X}^{2}}{s}\right)^{1-2 \alpha(0)}}{\left(1-\frac{M_{X}^{2}}{s}\right)^{n}} \frac{\gamma_{0}(t)}{1-\frac{M_{X}^{2}}{s}} \frac{\left(\frac{M_{X}^{2}}{s}\right)^{-2 \alpha^{\prime}(0) t}}{e^{b m_{D}^{2}+b\left(t-m_{D}^{2}\right)\left(1-\frac{M_{X}^{2}}{s}\right)}}
$$


where we have neglected the t/s contribution from eq.21 as explained in the appendix; $\alpha(0)$ refers to the $\mathrm{D}$ trajectory. For small $\mathrm{t}$, the first factor is dominant. For Table III, we find that our result for the power $\mathrm{n}$ is equal to 3.7 in good agreement with the experimental value from all three experiments. To show that the second factor in eq.14 does not disturb significantly the previous analysis, in Fig. 8 we plot $R / \gamma_{0}(t)$ for small and fixed t values. As expected no large variations are found.

\section{Possible dynamics for the phenomenological slope behaviour}

From our analysis it appears that the slope parameter depends on the constituent quark masses through a $3 / 2$ power law. In the following we present some simple considerations which may lead to such a behaviour.

It has already been shown [18], [19] that in a non relativistic approximation for the potential

$$
V(r)=a r^{p}
$$

$\mathrm{p}=2 / 3$ generates linear Regge trajectories. In such a model, the total energy is simply given by the centrifugal term and a confinement one, which is assumed to depend on the sum of the constituent masses $\left(\tilde{m}=m_{1}+m_{2}\right)$ through an arbitrary function, $a=a(\tilde{m})$

$$
E(r ; p)=\frac{J^{2}}{2 \Lambda r^{2}}+\frac{\Lambda(\Lambda r)^{p}}{p} a
$$

$\Lambda$ is a mass parameter and we require the centrifugal term to be $\tilde{m}$ independent in order to preserve a regular behaviour in the limit where the sum of the constituent masses vanish, i.e. the light mesonic sector. In such a model, a quasi linear $\mathrm{p}=2 / 3$ potential gives the phenomenological linear trajectories, when calculating the squared energy at the equilibrium point $r_{0}$

$$
r_{0}=\frac{1}{\Lambda}\left(\frac{J^{2}}{a}\right)^{3 / 8}
$$

Through a simple calculation, one can derive for the slope of the trajectory

$$
\alpha^{\prime}(\tilde{m})=\frac{1}{2 \Lambda^{2}} \frac{1}{a^{3 / 2}}
$$

In order to obtain our phenomenological result, eq.3, $a(\tilde{m})$ must be proportional to $\tilde{m}$ suggesting that a linear mass term is required in the confinement potential in order to obtain the $3 / 2$ power in the Regge slope. 


\section{Conclusions}

In the present work, we have approached the generic problem of hadron dynamics through considerations about the Regge trajectories for mesons of all flavors. Previously, Regge phenomenology has been succesfully applied in the light sector for both space- and time- like regions. The analysis of the Regge parameters for various flavors which we have performed does appear to justify the Regge technique as a powerful one.

As has been shown in section five, heavy flavour production from inclusive scattering is strongly suppressed with increasing energy. We succesfully checked our model predictions with available experimental data. Work is still in progress to investigate further how the bound states depend on flavour. An important task is to determine the relevant mass parameter describing the internal dynamics of the mesons. Until now, our choice of the sum of the constituent quark masses has been succesful. The phenomenological analysis does not show whether another combination of $m_{1}, m_{2}$ may also be relevant, yet, to this purpose, indirect applications of our results can be critically considered. Another interesting question is how to generate the observed mass dependence. A plausible choice, which reproduced the observerd phenomenology, has been discussed in section six, where a mass dependence was included in the confinement term.

\section{Acknowledgements}

S.F. would like to thank Professor S. Glashow and the Harvard Physics Department for their hospitality.

\section{References}

[1] F. Iachello, N.C.Mukhopadyay and L. Zhang Phys.Rev.D44 (1991), 898.

[2] Review of Particle Properties, Phys. Rev. D54 (1996),1-720.

[3] N.A.Tornqvist, in Stockholm 1990, Proceedings, Low energy antiproton physics,p. 287-303 and Helsinky University preprint HU-TFT-90-52.

[4] K.Johnson and C.B.Thorn, Phys. Rev.D13 (1976), 1934; I.Bars and A.J.Hanson, ibid. 13 (1976), 1744. For a review of the bag model see D. Flamm and F. Schoberl, Introduction to the quark model of elementary particles (Vol. I). Gordon and Breach Publishers(1982).

[5] J. L. Richardson, Phys. Lett. B82 (1979) 272.

[6] A. Martin, Phys. Lett. B 93 (1980) 338; B 100 (1981) 511.

[7] A. K. Grant, J. L. Rosner and E. Rynes, Phys. Rev. D 47 (1993) 1981. 
[8] J. Morishita, M. Kawaguchi and T. Morii, Phys. Lett. B185 (1987) 159; Phys. Rev. D37 (1988) 159.

[9] DELPHI Collaboration Phys. Lett. B398 (1997), 207; ALEPH Collaboration Phys.Lett. B402, 213.

[10] A.B.Kaidalov, Z. Phys. C12(1982),63;L.Burakovsky, L.P.Horwitz and T.Goldman hep-ph/9708468.

[11] S.Filipponi and Y. Srivastava, to be published; S.Filipponi, G.Pancheri and Y. Srivastava, Invited Talk at XXVII Symposium on Multiparticle Dynamics, (September 1997, LNF Frascati Rome), to be published.

[12] A. H. Muller, Phys. Rev. D2 (1970), 2963.

[13] G. Pancheri and Y. Srivastava, Lett. Nuovo Cimento Vol. II (1971) 381.

[14] Fermilab E769 Collaboration, G. A. Alves et al., Phys. Rev. Lett. 69 (1992) 3147; Phys. Rev. D 49 (1994) 4317.

[15] Na27 LEBC-EHS Collaboration, M. Aguillar Benitez et al., Phys. Lett. 161 B (1985), 400.

[16] Na32 ACCMOR Collaboration, S. Berlag et al., Z. Phys. C 49(1991), 555.

[17] ACCMOR Collaboration, S. Berlag et al. Phys. Lett. B 247 (1990) 113.

[18] A. Nakamura, G. Pancheri and Y. Srivastava Zeit.Phys. C21(1984), 243.

[19] J. Dias de Deus and J. Pulido Zeit.Phys. C9(1981), 255.

\section{Appendix}

\section{Kinematics of inclusive reactions}

For the inclusive reaction $a+b \rightarrow c+X$, the momentum variables for the a,b,c particles are defined as follows

$p_{a}=\left(E_{a}, 0,0, p\right) \quad p_{b}=\left(E_{b}, 0,0,-p\right) \quad p_{c}=\left(E, \vec{p}_{T}, p_{z}\right)$

The Feynman-x variable is $x_{F} \approx \frac{2 p_{z}}{\sqrt{s}}$.

In the large s kinematic limit (where $m_{a}$ and $m_{b}$ can be put to zero and $m_{c}^{2} / s$ neglected), the transformations between the $\left(x_{F}, p_{T}^{2}\right)$ and $\left(t, M_{X}^{2}\right)$ variables are

$$
\left\{\begin{array}{l}
x_{F}\left(t, M_{X}^{2}\right)=1-\frac{M_{X}^{2}}{s}+\frac{2 t}{s} \\
p_{T}^{2}\left(t, M_{X}^{2}\right)=-\left(t-m_{c}^{2}\right)\left(1-\frac{M_{X}^{2}}{s}+\frac{t}{s}\right)
\end{array}\right.
$$


Through the calculation of the Jacobian factor, the differential cross sections in terms of two sets of kinematic variables are related as follows

$$
\frac{d^{2} \sigma}{d x_{F} d p_{T}^{2}}=\left(1-\frac{M_{X}^{2}}{s}\right) \frac{d^{2} \sigma}{d t d M_{X}^{2}}
$$

Using the previous eqs. 19 and 20 (where now $\mathrm{a}=\pi^{-}, \mathrm{b}=N, \mathrm{c}=D$ ), the differential cross section, eq.12, for the $\pi^{-} N \rightarrow D X$ scattering in the $\left(M_{X}^{2}, t\right)$ variables is found to be

$$
\left[\frac{d^{2} \sigma}{d M_{X}^{2} d t}\right]_{E X P}=\frac{\left(1-\frac{M_{x}^{2}}{s}\right)}{s}\left(\frac{M_{x}^{2}}{s}-\frac{2 t}{s}\right)^{n} e^{b\left[m_{D}^{2}+\left(t-m_{D}^{2}\right)\left(1-\frac{M_{x}^{2}}{s}+\frac{t}{s}\right)\right]}
$$

Eq.21 depends on the variable $t$ and in the following we investigate whether the $\mathrm{t} / \mathrm{s}$ contribution can be neglected, in the tri-regge limit. For $m_{\pi}=m_{N}=0, \mathrm{t}$ is given by

$$
t\left(x_{F}, p_{T}^{2}\right)=m_{D}^{2}+x_{F} \frac{s}{2}\left(1-\sqrt{1+\frac{4}{s} \frac{m_{D}^{2}+p_{T}^{2}}{x_{F}^{2}}}\right)
$$

In Fig.9, we see that $\mathrm{t}$ runs from small to rather large values in the experimentally explored region, even for $p_{T}^{2}=0$. The figure refers to experiment E769, but the same result applies for all three experiments. For the tri-regge limit to be applicable, $\mathrm{t}$ has to be fixed and $M_{X}^{2} / s$ must be small. As a function of $x_{F}$ and $p_{T}^{2}, M_{X}^{2} / s$ is given by

$$
\frac{M_{X}^{2}}{s}=1+\frac{m_{D}^{2}}{s}-x_{F} \sqrt{1+\frac{4}{s} \frac{m_{D}^{2}+p_{T}^{2}}{x_{F}^{2}}} \approx 1-x_{F}
$$

so that the small $M_{X}^{2} / s$ condition requires large $x_{F}$. Returning to the t variable, we see that $\mathrm{t}$ is small at large $x_{F}$ and hence $t / s$ in eq.21 can be neglected.

Even though data are available up to $x_{F}^{\max }=0.7 \div 0.9$, but they are rather imprecise near the boundary, we limited our analysis to the reliable region $x_{F} \approx$ $0.4 \div 0.7\left(M_{X}^{2} / s \approx 0.3 \div 0.6\right)$ for testing our predictions. 


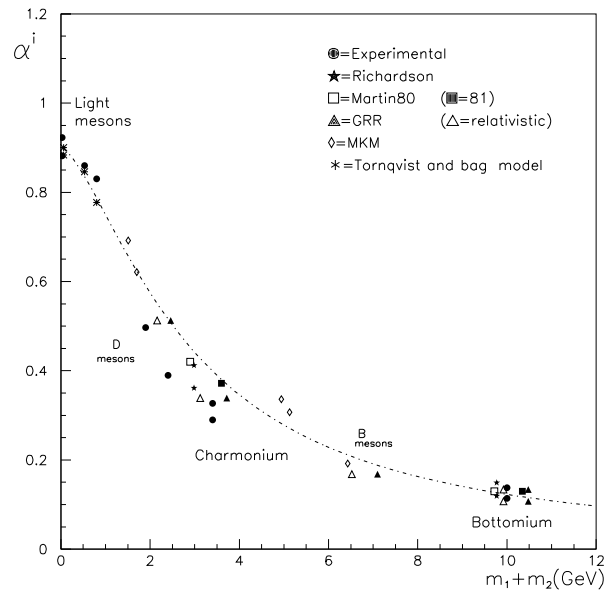

Figure 1: The slope parameter for various mesons as a function of $m_{1}+m_{2}$.

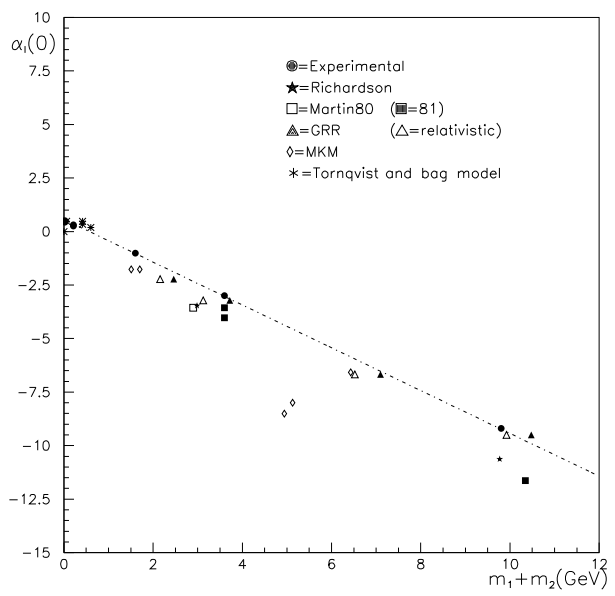

Figure 2: The $\alpha_{I}(0)$ parameter for various mesons as a function of $m_{1}+m_{2}$. 

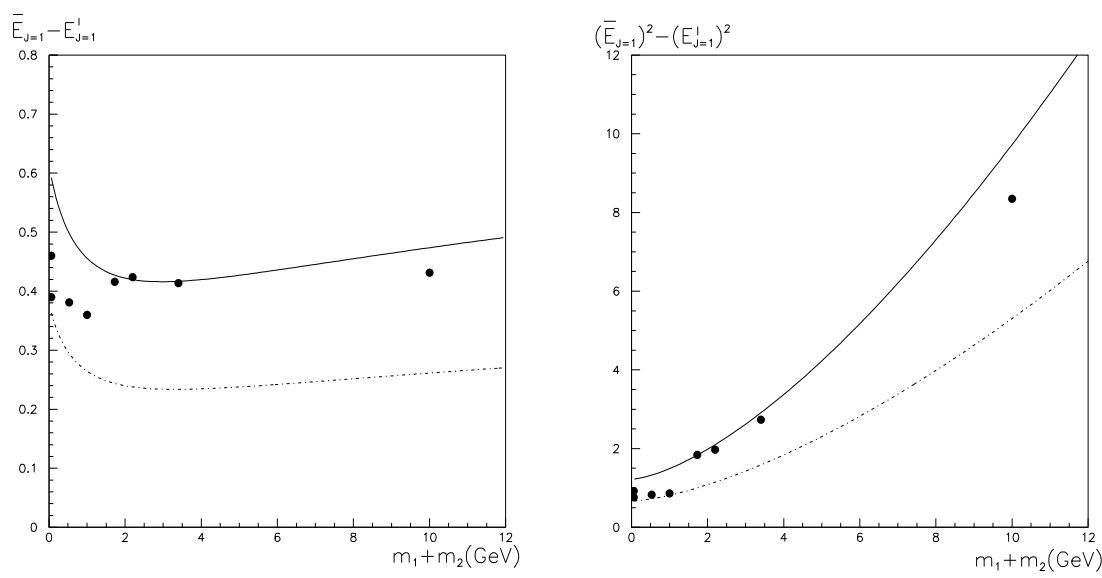

Figure 3: Left: The energy splitting between the $J^{P}=1^{-}$and $J^{P}=1^{+}$states of various mesons (in Table I) as a function of $m_{1}+m_{2}$. Right: Their energy squared gap. The curves are for $\bar{d} \alpha(0)=\alpha_{I}(0)-\bar{\alpha}(0)=0.6$ [down] and $\bar{d} \alpha(0)=$ 1.1 [up].

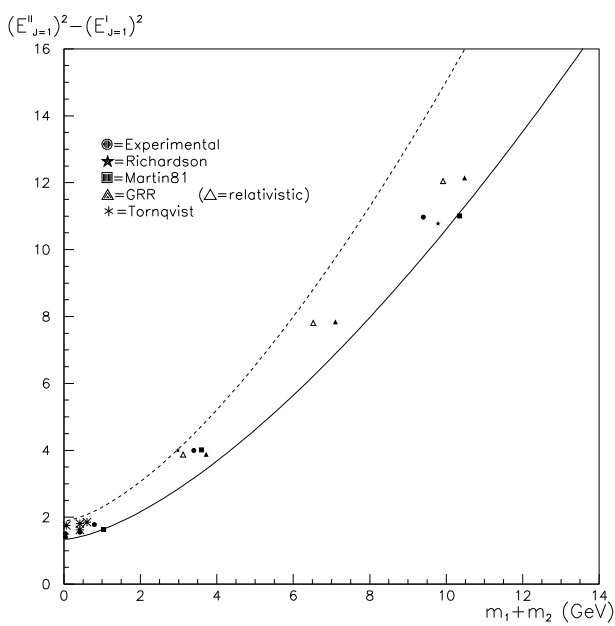

Figure 4: The energy squared gap between the $J=1$ states of the leading and second trajectory as a function of $m_{1}+m_{2}$. The curves are for $d \alpha(0)=\alpha_{I}(0)-$ $\alpha_{I I}(0)=1.3$ [down], and $d \alpha(0)=1.6[u p]$. 


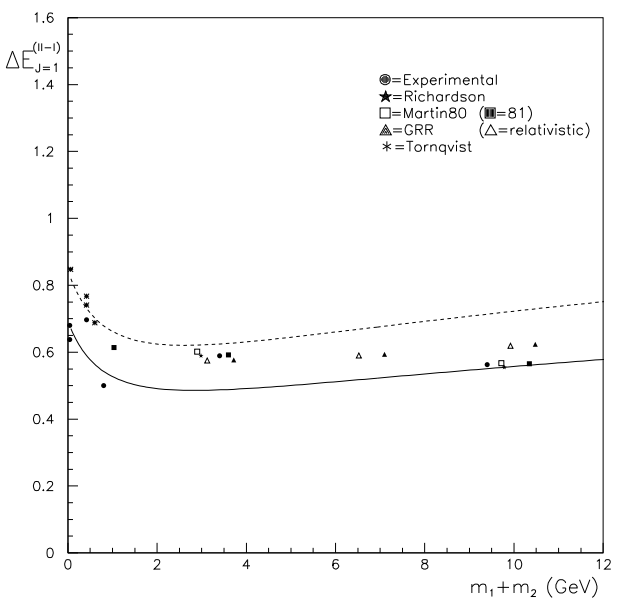

Figure 5: The energy splitting between the $J=1$ states of the leading and second trajectories as a function of $m_{1}+m_{2}$. The two curves are for $d \alpha(0)=\alpha_{I}(0)-$ $\alpha_{I I}(0)=1.3$ [down] and $d \alpha(0)=1.6$ [up].

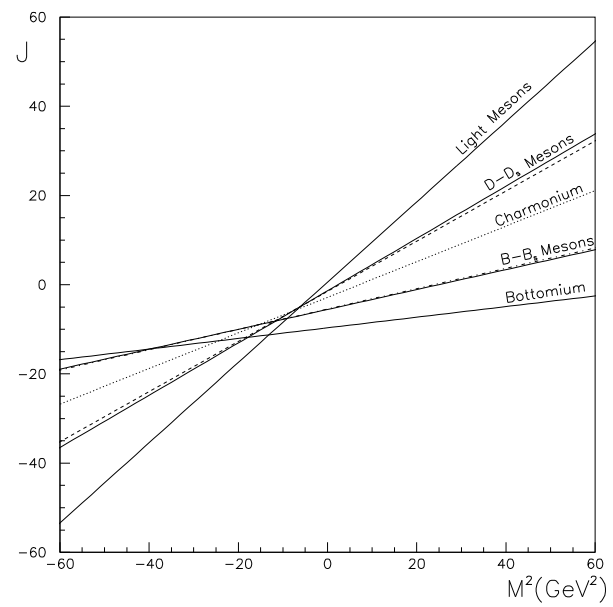

Figure 6: The leading Regge trajectories as derived by our model (see Table II) in a compared plot. 


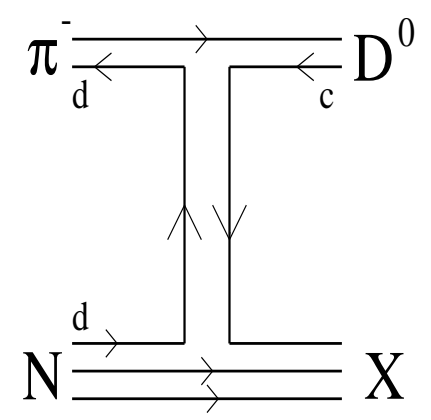

Figure 7: A D mesonic trajectory is exchanged in the $\pi^{-} N \rightarrow D X$ inclusive reaction.

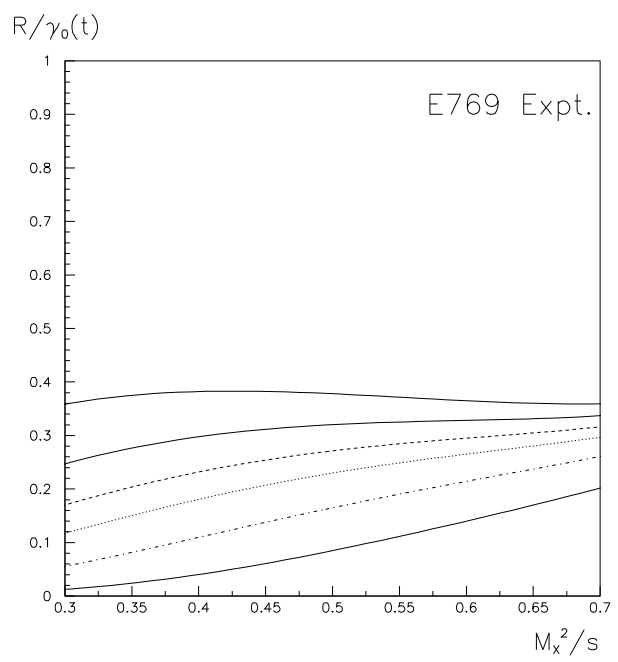

Figure 8: The $R / \gamma_{0}(t)$ function for fixed and small $t$ values. The $n$ and $b$ parameters are from E769 Expt. 


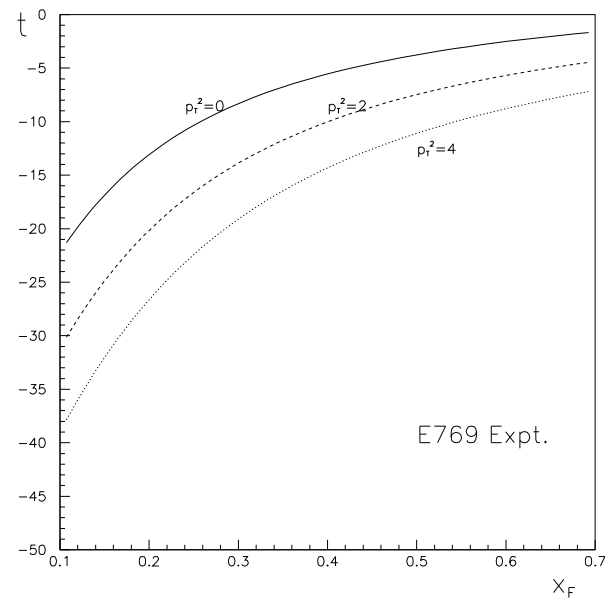

Figure 9: The $t\left(x_{F}\right)$ function for fixed $p_{T}^{2}$ values. Data are from the E769 Experiment. 